\section{Intervenção nutricional e prevenção primária do diabetes mellitus tipo 2: uma revisão sistemática}

\author{
Nutritional intervention and primary prevention \\ of type 2 diabetes mellitus: a systematic review
}

I Faculdade de Saúde
Pública, Universidade
de São Paulo,
São Paulo, Brasil.
2 Faculdade de Medicina
de Ribeirão Preto,
Universidade de São Paulo,
Ribeirão Preto, Brasil.
Correspondência
M. A. Cardoso
Departamento de Nutrição,
Faculdade de Saúde Pública,
Universidade de São Paulo.
Av. Dr. Arnaldo 715,
São Paulo, SP
o1246-904, Brasil.
marlyac@usp.br

Abstract

Type 2 diabetes mellitus is one of the most important public health problems in the world. Its increasing prevalence in several countries and the difficult metabolic control of individuals with the disease justify studying strategies for primary prevention. Recent randomized clinical trials suggest that nutritional intervention may have an important impact on risk reduction for type 2 diabetes mellitus among individuals at high risk. The present systematic review describes the effect of lifestyle intervention trials on primary prevention of type 2 diabetes mellitus.

Type II Diabetes Mellitus; Nutrition Therapy; Chronic Disease
Daniela Saes Sartorelli 1

Laércio Joel Franco 2

Marly Augusto Cardoso 1

\section{Introdução}

Em muitos países do mundo, a prevalência do diabetes mellitus tipo 2 tem se elevado vertiginosamente, e se espera um incremento ainda maior. Nos países em desenvolvimento, há uma tendência de aumento na freqüência em todas as faixas etárias, especialmente nas mais jovens, cujo impacto negativo sobre a qualidade de vida e a carga da doença aos sistemas de saúde é imensurável 1.

O controle metabólico de indivíduos com a doença em evolução consiste em um dos maiores desafios dos serviços de saúde pública americanos e do Brasil 2,3,4. Por isso, o desenvolvimento de programas eficazes e viáveis aos serviços públicos de saúde para a prevenção primária de diabetes mellitus tipo 2 em população de risco é necessário tanto para o controle de incidência de diabetes mellitus tipo 2 como também para a prevenção secundária de suas complicações metabólicas.

Segundo relatório recente da Organização Mundial da Saúde (OMS) sobre dieta, nutrição e prevenção de doenças crônicas não-transmissíveis (DCNT), a associação entre o ganho de peso, obesidade abdominal, sedentarismo e o desenvolvimento de diabetes mellitus tipo 2 é convincente, e o consumo alimentar habitual é considerado um dos principais fatores passíveis de modificação relacionados ao desenvolvimento de DCNT 5. 
O envelhecimento populacional e as alterações do estilo de vida são apontados como os principais determinantes do acentuado incremento na freqüência de diabetes mellitus tipo 2 nos últimos anos, o que pode ser fundamentado pela alarmante prevalência de alteração de homeostase glicêmica (AHG) entre indivíduos geneticamente susceptíveis expostos a drásticas mudanças do comportamento alimentar e prática de atividades físicas, como os índios Pima e migrantes de origem asiática residentes nos Estados Unidos e Brasil 1,6.

Evidências epidemiológicas provenientes de estudos prospectivos sugerem um efeito protetor para o diabetes mellitus tipo 2 por intermédio da adoção de um estilo de vida saudável. O Nurses Health Study, conduzido com 84.941 mulheres americanas, observou que a ausência de tabagismo, prática de trinta minutos de atividades físicas diária, manutenção de peso e padrão alimentar habitual rico em fibras e ácidos graxos polinsaturados, pobre em gorduras saturadas e ácidos graxos trans com baixo índice glicêmico reduziu em $91,0 \%$ o risco de desenvolver o diabetes mellitus tipo 2 após 16 anos de seguimento 7 .

Um ensaio clínico não aleatório com intervenção no estilo de vida, Malmö Preventive Trial 8 , conduzido entre 222 adultos, demonstrou que mortalidade de indivíduos portadores de tolerância à glicose diminuída (TDG) $(6,5 /$ 1.000 pessoas ao ano) que receberam orientações nutricionais durante os seis primeiros anos de seguimento foi semelhante ao observado entre os normoglicêmicos (6,2/1.000 pessoas ao ano) e inferior ao registrado entre indivíduos com TGD que não participaram da intervenção nutricional (14,0/1.000 pessoas ao ano) após 12 anos de seguimento, sugerindo um impacto positivo da orientação do estilo de vida de indivíduos com AHG. Entretanto, a alocação não aleatória dos indivíduos entre os grupos de tratamento limita a interpretação de seus resultados.

Segundo a OMS, o número de estudos prospectivos e ensaios clínicos aleatórios de intervenção nutricional para a prevenção de diabetes mellitus tipo 2 ainda não é suficiente na demonstração de sua força causal 5 . Assim, o presente artigo de revisão descreve os resultados de ensaios clínicos aleatórios controlados para prevenção primária de diabetes mellitus tipo 2 conduzidos em adultos portadores de fatores de risco, cuja intervenção constou de orientação nutricional.

\section{Métodos}

Para a seleção dos artigos adotou-se a técnica de revisão sistemática com o objetivo de identificar ensaios clínicos aleatórios controlados de intervenção nutricional para a prevenção primária do diabetes mellitus tipo 2 em adultos publicados entre janeiro de 1995 a janeiro de 2005. Foram selecionados os estudos de prevenção do diabetes conduzidos em indivíduos com idade $\geq 19$ anos não institucionalizados, que relataram: (1) alocação aleatória dos grupos; (2) pelo menos um dos grupos constava de intervenção nutricional e pelo menos um grupo controle; (3) avaliação de peso corporal, consumo alimentar e glicemia de jejum antes e após o período de intervenção. Foram excluídos os estudos conduzidos entre indivíduos: (1) com diagnóstico prévio de diabetes, doenças cardiovasculares ou câncer; (2) em uso de drogas que alteram o metabolismo de carboidratos ou lipídeos; (3) gestantes ou nutrizes.

A pesquisa foi realizada nas bases de dados do MEDLINE e Literatura Latino-americana e do Caribe de Informação em Ciências da Saúde (LILACS) com as palavras-chave em inglês no resumo: "randomized controlled trials", "nutrition", "diet", “nutritional intervention”, "lifestyle intervention" associadas aos termos "prevention" e "diabetes". Apesar dos termos-chave utilizados em inglês para a busca de resumos, incluímos na pesquisa artigos publicados em português ou espanhol.

\section{Resultados}

Dos 162 artigos identificados mediante as palavras-chave empregadas, nove estudos publicados atendiam a todos os critérios de inclusão estabelecidos. As informações sobre população, local de estudo, programa de intervenção nutricional e principais desfechos dos ensaios clínicos aleatórios selecionados estão apresentadas de forma sucinta na Tabela 1. Para os estudos com tamanho amostral e tempo de seguimento com poder de avaliar a redução de incidência de diabetes mellitus tipo 2, atribuímos como desfecho primário o impacto na ocorrência da doença entre os indivíduos que receberam orientações nutricionais. Nos estudos com objetivo de avaliar o impacto da intervenção no controle de fatores de risco para o diabetes mellitus tipo 2, as alterações observadas de glicemia e de perfil de lipídios séricos entre os indivíduos do grupo "intervenção" foram consideradas como desfechos primários, definindo-se como desfechos secundários as alterações de peso e estilo de vida. 
Descrição de ensaios clínicos aleatorizados com intervenção nutricional para a prevenção primária do diabetes mellitus tipo 2 .

\begin{tabular}{|c|c|c|c|c|c|c|c|}
\hline Referências & Data & $\begin{array}{l}\text { População } \\
\text { de estudo }\end{array}$ & Local (duração) & $\begin{array}{l}\text { Grupos } \\
\text { de estudo }\end{array}$ & $\begin{array}{l}\text { Programa de } \\
\text { intervenção }\end{array}$ & $\begin{array}{l}\text { Desfechos } \\
\text { primários }\end{array}$ & $\begin{array}{l}\text { Desfechos } \\
\text { secundários }\end{array}$ \\
\hline \multicolumn{8}{|l|}{$\begin{array}{l}\text { Estudos } \\
\text { conduzidos } \\
\text { em países } \\
\text { desenvolvidos }\end{array}$} \\
\hline Wing et al. 9 & 1998 & $\begin{array}{l}\mathrm{n}=154 \\
80,0 \% \text { do sexo } \\
\text { feminino } \\
\text { Idade média: } \\
45 \pm 4,5 \text { anos } \\
\mathrm{N}, \mathrm{GJA} \text { ou TGD* } \\
\text { História familiar } \\
\text { de diabetes } \\
\text { mellitus do tipo } 2^{*} \\
\text { IMC médio: } \\
36 \pm 4,0 \mathrm{~kg} / \mathrm{m}^{2}\end{array}$ & $\begin{array}{l}\text { Estados Unidos } \\
\text { (2 anos) }\end{array}$ & $\begin{array}{l}\text { 1. Dieta }(n=37) \\
\text { 2. Dieta+exer- } \\
\text { cícios }(n=40) \\
\text { 3. Exercícios } \\
(n=37) \\
\text { 4. Controle } \\
(n=40)\end{array}$ & $\begin{array}{l}\text { 1. Dieta: } \\
\downarrow \text { calorias (800- } \\
\text { 1.200kcal/dia) } \\
\downarrow \text { gorduras da } \\
\text { dieta (<20,0\% } \\
\text { das calorias) } \\
\text { 2. Exercícios: } \\
\text { 50-60min/dia } \\
\text { de atividades } \\
\text { vigorosas (gasto } \\
\text { energético de } \\
\text { 1.500kcal/semana) } \\
\text { 3. Dieta+ } \\
\text { exercícios: } \\
\text { Ambos os } \\
\text { programas }\end{array}$ & $\begin{array}{l}\text { Após 6, } 12 \text { e } 24 \\
\text { meses } \\
\text { Incidência do } \\
\text { diabetes mellitus } \\
\text { tipo } 2^{\star \star} \\
\text { Glicemia de } \\
\text { jejum** }\end{array}$ & $\begin{array}{l}\text { Após } 6 \text { e } 12 \text { meses } \\
\text { 1. Dieta: } \\
\downarrow 9,0 \% \text { e } 5,0 \% \text { peso } \\
\downarrow 10,0 \% \text { LDL-c** } \\
\text { 2. Exercícios: } \\
\downarrow 2,0 \% \text { e peso** } \\
\text { LDL-c** } \\
\text { 3. Dieta+ } \\
\text { exercícios: } \\
\downarrow 10,0 \% \text { e } \\
\text { 7,0\% peso } \\
\text { LDL-c ** }\end{array}$ \\
\hline Wein et al 11 & 1999 & $\begin{array}{l}\mathrm{n}=200 \\
100,0 \% \text { do sexo } \\
\text { feminino } \\
\text { Idade média: } \\
38 \pm 3,9 \text { anos } \\
\text { TGD } \\
\text { Diabetes mellitus } \\
\text { tipo } 2 \text { gestacional } \\
\text { prévio } \\
\text { IMC médio: } \\
25,4 \pm 2,7 \mathrm{~kg} / \mathrm{m}^{2}\end{array}$ & Austrália (4 anos) & $\begin{array}{l}\text { 1. Dieta }(n=100) \\
\text { 2. Controle } \\
(n=100)\end{array}$ & $\begin{array}{l}\text { 1. Dieta: } \\
\text { Orientações } \\
\text { gerais sobre } \\
\text { alimentação } \\
\text { saudável } \\
\uparrow \text { fibras } \\
\downarrow \text { gorduras e } \\
\text { açúcares } \\
90 \text { atividades } \\
\text { físicas/semana }\end{array}$ & $\begin{array}{l}\text { Após } 4 \text { anos } \\
\text { Incidência do } \\
\text { diabetes mellitus } \\
\text { tipo } 2^{\star \star} \\
\text { Glicemia de } \\
\text { jejum** } \\
\text { Glicemia após } \\
2 \text { horas** }\end{array}$ & $\begin{array}{l}\text { Após } 4 \text { anos } \\
\text { 1. Dieta: } \\
\text { 3,0\% IMC } \\
\text { Exercícios** } \\
\text { Gordura da dieta** } \\
\text { Açúcares da dieta** }\end{array}$ \\
\hline Tuomilehto et al. 13 & 2001 & $\begin{array}{l}\mathrm{n}=522 \\
57,0 \% \text { do sexo } \\
\text { feminino } \\
\text { Idade média: } \\
55 \pm 7 \text { anos } \\
\text { TGD } \\
\text { IMC médio: } \\
31,2 \pm 4,2 \mathrm{~kg} / \mathrm{m}^{2}\end{array}$ & Finlândia (3 anos) & $\begin{array}{l}\text { 1. Estilo de vida } \\
(n=265) \\
\text { 2. Controle } \\
(n=257)\end{array}$ & $\begin{array}{l}\text { 1. Estilo de vida: } \\
\downarrow \text { gorduras } \\
\text { (<30,0\%), } \\
\text { saturadas } \\
(<10,0 \%) \\
\text { fibras (>15g/ } \\
1.000 \mathrm{kcal} / \mathrm{dia}) \\
210 \mathrm{~min} / \mathrm{sem} \text { de } \\
\text { atividades físicas }\end{array}$ & $\begin{array}{l}\text { 1.Estilo de vida: } \\
\text { Após } 3 \text { anos } \\
\downarrow 58,0 \% \text { da } \\
\text { incidência do } \\
\text { diabetes mellitus } \\
\text { tipo } 2 \text { no grupo } \\
\text { estilo de vida }\end{array}$ & $\begin{array}{l}\text { 1. Estilo de vida: } \\
\text { Após } 1 \text { ano } \\
\downarrow 2,0 \% \text { colesterol } \\
\text { total } \\
\downarrow 11,6 \% \\
\text { triglicérides } \\
\downarrow 3,5 \% \text { PAS } \\
\text { Após } 3 \text { anos } \\
\downarrow 4,7 \% \text { peso } \\
\text { corporal } \\
\downarrow 4,3 \% \text { cintura }\end{array}$ \\
\hline $\begin{array}{l}\text { Diabetes } \\
\text { Prevention } \\
\text { Program } 12\end{array}$ & 2002 & $\begin{array}{l}\mathrm{n}=3.234 \\
70,0 \% \text { do sexo } \\
\text { feminino } \\
\text { Idade média: } \\
50,6 \pm 10,7 \text { anos } \\
\text { TGD } \\
\text { IMC médio: } \\
34,0 \pm 6,7 \mathrm{~kg} / \mathrm{m}^{2}\end{array}$ & $\begin{array}{l}\text { Estados Unidos } \\
\text { (3 anos) }\end{array}$ & $\begin{array}{l}\text { 1. Estilo de vida } \\
(\mathrm{n}=1.079) \\
\text { 2. Metformina } \\
(\mathrm{n}=1.073) \\
\text { 3. Controle/ } \\
\text { placebo } \\
(\mathrm{n}=1.082)\end{array}$ & $\begin{array}{l}\text { 1. Estilo de vida: } \\
\downarrow \text { calorias } \\
\downarrow \text { gorduras totais } \\
\text { (<25,0\%) } \\
\text { Orientação } \\
\text { pirâmide alimen- } \\
\text { tar americana } \\
\text { 150min de } \\
\text { atividades físi- } \\
\text { cas/semana } \\
\text { 2. Metformina: } \\
850 \text { mg metformina } \\
2 \text { vezes ao dia }\end{array}$ & $\begin{array}{l}\text { Após } 3 \text { anos } \\
\text { 1. Estilo de vida: } \\
\downarrow 58,0 \% \text { incidên- } \\
\text { cia do diabetes } \\
\text { mellitus tipo } 2 \\
\text { 2. Metformina: } \\
\downarrow 30,0 \% \text { incidên- } \\
\text { cia do diabetes } \\
\text { mellitus tipo } 2\end{array}$ & $\begin{array}{l}\text { Após } 3 \text { anos } \\
\text { 1. Estilo de vida: } \\
\downarrow 6,0 \% \text { peso } \\
\text { corporal } \\
2 \text {. Metformina: } \\
\downarrow 2,1 \mathrm{~kg} \text { peso }\end{array}$ \\
\hline
\end{tabular}




\begin{tabular}{|c|c|c|c|c|c|c|c|}
\hline Referências & Data & $\begin{array}{l}\text { População } \\
\text { de estudo }\end{array}$ & Local (duração) & $\begin{array}{l}\text { Grupos } \\
\text { de estudo }\end{array}$ & $\begin{array}{l}\text { Programa de } \\
\text { intervenção }\end{array}$ & $\begin{array}{l}\text { Desfechos } \\
\text { primários }\end{array}$ & $\begin{array}{l}\text { Desfechos } \\
\text { secundários }\end{array}$ \\
\hline McAuley et al. 16 & 2002 & $\begin{array}{l}\mathrm{n}=79 \\
67,0 \% \text { do sexo } \\
\text { feminino } \\
\text { Idade: } 30-68 \text { anos } \\
\text { Resistência } \\
\text { a insulina } \\
\text { IMC médio: } \\
34,3 \pm 4,4 \mathrm{~kg} / \mathrm{m}^{2}\end{array}$ & $\begin{array}{l}\text { Nova Zelândia } \\
\text { (4 meses) }\end{array}$ & $\begin{array}{l}\text { 1. Estilo de vida } \\
\text { moderado }(n=31) \\
\text { 2. Estilo de vida } \\
\text { intenso }(n=25) \\
\text { 3. Controle } \\
(n=23)\end{array}$ & $\begin{array}{l}\text { 1. Estilo de vida } \\
\text { moderado: } \\
<32,0 \% \text { gordura } \\
\text { (<11,0\% satura- } \\
\text { das, } 14,0 \% \text { mono- } \\
\text { insaturados, 7,0\% } \\
\text { polinsaturadas) } \\
<200 \mathrm{mg} \text { coles- } \\
\text { terol/dia } \\
\text { fibras (> 25g/dia) } \\
\text { 150min de ativi- } \\
\text { dades físicas mo- } \\
\text { derada/semana } \\
2 . \text { Estilo de vida } \\
\text { intenso } \\
<26,0 \% \text { gordura } \\
\text { (<6,0\% saturadas, } \\
\text { 13,0\% monoinsa- } \\
\text { turados, 7,0\% } \\
\text { polinsaturadas) } \\
<140 \mathrm{mg} \text { coles- } \\
\text { terol/dia } \\
\text { fibras (> 35g/dia) } \\
150 \mathrm{~min} \text { de ativi- } \\
\text { dades físicas } \\
\text { intensa/semana }\end{array}$ & 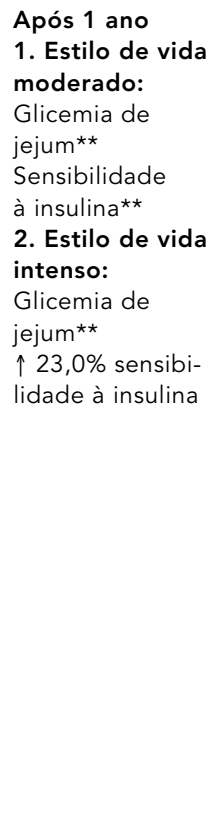 & $\begin{array}{l}\text { Após } 1 \text { ano } \\
\text { 1. Estilo de vida } \\
\text { moderado: } \\
\downarrow 5,4 \% \text { peso } \\
\text { corporal } \\
\text { Resistência física*^ } \\
\downarrow \text { gordura dieta } \\
\text { 2. Estilo de vida } \\
\text { intenso: } \\
\downarrow 6,9 \% \text { peso } \\
\text { corporal } \\
11,0 \% \text { resistência } \\
\text { física } \\
\downarrow \text { gordura dieta }\end{array}$ \\
\hline Watanabe et al. 17 & 2002 & $\begin{array}{l}\mathrm{n}=173 \\
100,0 \% \text { do sexo } \\
\text { masculino } \\
\text { Idade média: } \\
55 \pm 7,0 \text { anos } \\
\text { Glicemia } 1 \mathrm{~h}: \\
180-200 \mathrm{mg} / \mathrm{dl} \\
\text { IMC médio: } \\
24,4 \pm 2,9 \mathrm{~kg} / \mathrm{m}^{2}\end{array}$ & Japão (1 ano) & $\begin{array}{l}\text { 1. Dieta }(n=79) \\
\text { 2. Controle } \\
(n=77)\end{array}$ & $\begin{array}{l}\text { 1. Dieta: } \\
\downarrow \text { calorias } \\
\text { do jantar } \\
20,0-25,0 \% \\
\text { gordura } \\
\uparrow \text { cereais integrais, } \\
\text { vegetais, frutas, } \\
\text { laticínios desna- } \\
\text { tados, peixes } \\
\downarrow \text { carnes e ovos }\end{array}$ & $\begin{array}{l}\text { Após } 1 \text { ano } \\
1 \text {. Dieta } \\
\downarrow 8,0 \% \text { glicemia } \\
2 \mathrm{hs}\end{array}$ & $\begin{array}{l}\text { Após } 1 \text { ano } \\
\text { 1. Dieta } \\
\downarrow 3,0 \% \text { calorias } \\
\text { do jantar }\end{array}$ \\
\hline Mensink et al. 18 & 2003 & $\begin{array}{l}\mathrm{n}=102 \\
43,0 \% \text { do sexo } \\
\text { feminino } \\
\text { Idade média: } \\
56 \pm 1 \text { ano } \\
\text { TGD } \\
\text { IMC médio: } \\
29,5 \pm 0,5 \mathrm{~kg} / \mathrm{m}^{2}\end{array}$ & Holanda (1 ano) & $\begin{array}{l}\text { 1. Estilo de vida } \\
(n=47) \\
\text { 2. Controle } \\
(n=55)\end{array}$ & $\begin{array}{l}1 . \text { Estilo de vida } \\
<10,0 \% \text { gordu- } \\
\text { ras saturadas } \\
<80 \mathrm{mg} \text { coleste- } \\
\text { rol dietético/ } \\
1.000 \mathrm{kcal} / \mathrm{dia} \\
\geq 7 \mathrm{~g} \mathrm{de} \mathrm{fibras} \\
\text { totais / } \\
1.000 \mathrm{kcal} / \mathrm{dia} \\
150 \text { minutos de } \\
\text { atividades físicas / } \\
\text { semana }\end{array}$ & $\begin{array}{l}\text { Após } 1 \text { ano } \\
1 \text {. Estilo de vida } \\
\downarrow 13,0 \% \text { glice- } \\
\text { mia } 2 \text { hs } \\
\downarrow 19,0 \% \text { HOMA } \\
\text { Glicemia jejum** }\end{array}$ & $\begin{array}{l}\text { Após } 1 \text { ano } \\
1 \text {. Estilo de vida } \\
\downarrow 3,0 \% \text { peso } \\
\text { corporal } \\
\downarrow \text { calorias, gordura } \\
\text { dieta } \\
\uparrow \text { fibras }\end{array}$ \\
\hline
\end{tabular}

Ensaios clínicos aleatorizados controlados de intervenção nutricional

Um dos ensaios clínicos aleatórios controlados pioneiros conduzidos nos Estados Unidos com o objetivo de avaliar o impacto da intervenção no estilo de vida na incidência do diabetes mellitus tipo 2 foi publicado por Wing et al. no ano de 1998 9. Os 154 adultos, recrutados por intermédio de anúncios em jornais, portadores de obesidade e história familiar de diabetes mellitus tipo 2 foram aleatoriamente distribuídos entre os grupos "controle", "dieta”, "dieta e exercícios” ou "exercícios físicos”. O programa de intervenção constou de orientações em grupo com freqüência semanal durante os seis primeiros meses e reuniões quinzenais durante os dois anos de seguimento. As consultas semanais dos indivíduos do grupo "exercícios" constaram da prática supervisionada de caminha- 
Tabela 1 (continuação)

\begin{tabular}{|c|c|c|c|c|c|c|c|}
\hline Referências & Data & $\begin{array}{l}\text { População } \\
\text { de estudo }\end{array}$ & Local (duração) & $\begin{array}{l}\text { Grupos } \\
\text { de estudo }\end{array}$ & $\begin{array}{l}\text { Programa de } \\
\text { intervenção }\end{array}$ & $\begin{array}{l}\text { Desfechos } \\
\text { primários }\end{array}$ & $\begin{array}{l}\text { Desfechos } \\
\text { secundários }\end{array}$ \\
\hline \multicolumn{8}{|c|}{$\begin{array}{l}\text { Estudos conduzidos } \\
\text { em países em } \\
\text { desenvolvimento }\end{array}$} \\
\hline Pan et al. 10 & 1997 & $\begin{array}{l}n=577 \\
47,0 \% \text { do sexo } \\
\text { feminino } \\
\text { idade média: } \\
45 \pm 9,1 \text { anos } \\
\text { TGD } \\
\text { IMC médio: } \\
25,8 \pm 3,8 \mathrm{~kg} / \mathrm{m}^{2}\end{array}$ & China (6 anos) & $\begin{array}{l}\text { 1. Dieta }(n=130) \\
\text { 2. Exercícios } \\
(n=141) \\
\text { 3. Dieta+exercí- } \\
\text { cios }(n=126) \\
\text { 4. Controle } \\
(n=133)\end{array}$ & $\begin{array}{l}\text { 1. Dieta: } \\
\downarrow \text { calorias (20- } \\
25 \mathrm{kcal} / \mathrm{kg} \text { ) entre } \\
\text { indivíduos com } \\
\mathrm{IMC}>25 \mathrm{~kg} / \mathrm{m}^{2} \\
\downarrow \text { gorduras totais } \\
(<30,0 \% \text { ), álcool } \\
\text { e açúcar simples } \\
\text { verduras e } \\
\text { legumes } \\
\text { 2. Exercícios: } \\
210 \mathrm{~min} / \mathrm{semanais} \\
\text { de atividades } \\
\text { leves ou } 140 \mathrm{~min} / \\
\text { semanais de } \\
\text { atividades } \\
\text { moderadas ou } \\
70 \text { min/semanais } \\
\text { de atividades } \\
\text { intensas } \\
\text { 3. Dieta + } \\
\text { exercício: } \\
\text { Ambos os } \\
\text { programas }\end{array}$ & $\begin{array}{l}\text { Após } 6 \text { anos } \\
\text { 1. Dieta } \\
\downarrow 31,0 \% \text { incidên- } \\
\text { cia do diabetes } \\
\text { mellitus tipo } 2^{*} \\
2 \text {. Exercícios: } \\
\downarrow 46,0 \% \text { incidên- } \\
\text { cia do diabetes } \\
\text { mellitus tipo } 2^{*} \\
\text { 3. Dieta+ } \\
\text { Exercícios: } \\
\downarrow 42,0 \% \text { incidên- } \\
\text { cia do diabetes } \\
\text { mellitus tipo } 2^{*}\end{array}$ & $\begin{array}{l}\text { Após } 6 \text { anos } \\
\text { 1. Dieta: } \\
\downarrow 3,6 \% \text { IMC** } \\
\text { 2. Exercícios: } \\
\downarrow 0,9 \% \text { IMC } \\
\text { 3. Dieta+ } \\
\text { Exercícios: } \\
\downarrow 1,8 \% \text { IMC }\end{array}$ \\
\hline Sartorelli et al. 20 & 2004 & $\begin{array}{l}n=104 \\
80,0 \% \text { do sexo } \\
\text { feminino } \\
\text { idade média: } \\
46 \pm 9,5 \text { anos } \\
\mathrm{N}, \mathrm{GJA}, \mathrm{TGD} \\
\text { IMC médio: } \\
28,8 \pm 2,5 \mathrm{~kg} / \mathrm{m}^{2}\end{array}$ & Brasil (1 ano) & $\begin{array}{l}\text { 1. Estilo de vida } \\
(n=51) \\
\text { 2. Controle } \\
(n=53)\end{array}$ & $\begin{array}{l}\text { 1. Estilo de vida: } \\
\uparrow \text { frutas, verduras, } \\
\text { legumes, cereais } \\
\text { integrais, gorduras } \\
\text { monoinsaturadas } \\
\downarrow \text { carnes e } \\
\text { laticínios inte- } \\
\text { grais, gorduras } \\
\text { saturadas, e } \\
\text { açúcar simples } \\
30 \text { minutos de } \\
\text { atividades físi- } \\
\text { cas/dia }\end{array}$ & $\begin{array}{l}\text { Após } 6 \text { e } 12 \\
\text { meses } \\
\text { 1. Estilo de vida: } \\
\downarrow 12,0 \% \text { e coles- } \\
\text { terol total** } \\
\downarrow 15,0 \% \text { e } \\
15,0 \% \text { LDL-c } \\
\text { HDL-c** } \\
\text { Glicemia de } \\
\text { jejum }{ }^{\star \star}\end{array}$ & $\begin{array}{l}\text { Após } 6 \text { e } 12 \text { meses } \\
1 \text {. Estilo de vida: } \\
\downarrow 3,1 \% \text { e } 3,4 \% \text { peso } \\
\downarrow \text { gordura saturada } \\
\text { frutas, verduras, } \\
\text { azeite de oliva } \\
\text { Atividade física** }\end{array}$ \\
\hline
\end{tabular}

* conforme critérios da OMS (1998);

** não foram observadas diferenças estatisticamente significantes entre os grupos.

$\mathrm{N}=$ glicemia normal; GJA = glicemia de jejum alterada; TGD = tolerância à glicose diminuída; IMC = índice de massa corporal (kg/m2);

LDL-c = lipoproteína de baixa densidade (low density lipoprotein, em inglês); PAS = pressão arterial sistólica (mmHg);

HOMA = indicador de resistência à insulina (homeostasis model assessment, em inglês); HDL-c = lipoproteína de alta densidade

(high density lipoprotein, em inglês).

das. Todos os participantes foram submetidos à avaliação clínica, bioquímica, antropométrica e de estilo de vida no início do estudo e após 6,12 e 24 meses com perdas de seguimento de $15,0,22,0$ e $26,0 \%$, respectivamente. Os autores não relataram as metas de adesão estabelecidas pelo programa.

Os principais resultados do estudo de Wing et al. 9 demonstraram que as médias (DP) de redução de peso corpóreo após seis meses de seguimento foram mais expressivas nos grupos “dieta” $[-9,1(6,4) \mathrm{kg}]$ e "dieta+exercícios" [-10,3 $(7,7) \mathrm{kg}$ ] quando comparadas aos grupos "exer- cícios" [-2,1 (4,2)kg] e "controle" [-1,5 (2,7)kg], segundo teste de Kruskal-Wallis $(p<0,05)$. Entretanto, a redução de peso após 12 meses de seguimento manteve-se estatisticamente significante apenas entre indivíduos dos grupos "dieta" [-5,5 (6,9)kg] e "dieta+exercícios" [-7,4 $(9,7) \mathrm{kg}$ ] (teste $t$ de Student). Após 24 meses, não houve manutenção das alterações de peso. Em relação às alterações do perfil de lipídios séricos, após seis meses de seguimento, houve reduções estatisticamente significantes de LDL colesterol (LDL-c) apenas entre os indivíduos do grupo "dieta” [-12,5 (2,3) mg/dl]. Entretanto, 
esta diferença não se manteve após 12 e 24 meses de seguimento. Nenhuma diferença estatisticamente significante foi observada para valores de glicemia e incidência de diabetes mellitus tipo 2 entre os grupos. Em relação ao consumo alimentar, houve uma redução média (DP) de $5,2(8,7)$ e $6,4(9,9)$ do percentual das calorias totais $(\% \mathrm{CT})$ provenientes de lipídios nos grupos "dieta" e "dieta e exercícios", respectivamente, após seis meses de seguimento. Após 24 meses de seguimento, houve manutenção do menor consumo de lipídios; porém, esta alteração foi semelhante ao relatado pelos participantes do grupo controle [redução de 4,3 $(7,2) \%$ CT de lipídios] em relação ao início do estudo.

Um ensaio clínico aleatório controlado, $D a$ Qing IGT and Diabetes Study 10, conduzido na China, avaliou o impacto da intervenção nutricional na incidência de diabetes mellitus tipo 2 entre adultos. Os participantes do estudo foram selecionados após avaliação de saúde populacional em uma cidade industrial. A população de estudo constou de 577 adultos portadores de excesso de peso e TGD, sendo aleatoriamente alocada em clínicas de tratamento: "dieta", "exercícios", "dieta e exercícios", além do grupo "controle”. Os programas de intervenção constaram de orientações individualizadas e sessões em grupo com freqüência semanal durante o primeiro mês, mensal nos próximos três meses e trimestral até o sexto ano de seguimento. Todos os participantes foram submetidos a exames clínicos, bioquímicos, antropométricos e de estilo de vida no princípio do estudo e após seis anos de seguimento com perda de seguimento de $8,0 \%$. Os autores não relataram as metas estabelecidas pelo programa.

Os principais resultados do estudo chinês 10 demonstraram que a incidência do diabetes mellitus tipo 2 (100 pessoas/ano) com intervalo de $95 \%$ de confiança (IC95\%) nos grupos "exercícios" $[5,1(2,6-7,6)]$ e "dieta e exercícios" $[6,8(3,6-10,0)]$ foi estatisticamente diferente quando comparada ao grupo controle $[13,3$ $(8,9-17,7)]$ após seis anos de seguimento. Os indivíduos do grupo "dieta e exercícios” relataram um aumento médio (DP) de 0,9 $(0,1)$ unidades/dia ( 1 unidade por dia $=30$ minutos de atividades físicas leves ou 20 minutos de atividades físicas moderadas ou 10 minutos de atividades físicas intensas) de prática de atividades físicas após seis anos de seguimento. Não ocorreram alterações de peso ou consumo alimentar habitual entre os participantes do estudo. Apesar das limitações referentes à aleatorização por clínicas de tratamento, este estudo forneceu evidências da eficácia de programas de estilo de vida de baixo custo na redução da incidência de diabetes mellitus tipo 2.

Na Austrália, um ensaio clínico aleatorizado controlado foi conduzido entre duzentas mulheres adultas de origem multiétnica portadoras de TGD e história pregressa de diabetes mellitus tipo 2 gestacional com o objetivo de avaliar o impacto de orientações gerais de alimentação saudável fornecidas por telefone na incidência do diabetes mellitus tipo 211 . As participantes do estudo foram selecionadas após exames de rotina realizados em um hospital em Melbourne, sendo aleatoriamente alocadas entre os grupos "controle" e "dieta”, cujo programa constou de orientações escritas sobre alimentação saudável e prática de atividades físicas e contatos telefônicos com a nutricionista com freqüência trimestral. Todas as participantes foram submetidas à avaliação clínica, bioquímica, antropométrica e de estilo de vida no início do estudo, anualmente e após quatro anos de seguimento com perda final de 3,5\%. Após quatro anos de seguimento, apesar da normalização da glicemia em $44,0 \%$ das participantes do estudo, não houve diferença na incidência anual do diabetes mellitus tipo 2 entre os grupos intervenção $(6,1 \%)$ e controle $(7,3 \%)$, com uma razão de taxa de incidência (IC95\%) de $0,83(0,47-1,48)$. Não houve diferença entre os grupos para o consumo alimentar habitual e prática de atividades físicas, observando-se um incremento estatisticamente significante para os valores de índice de massa corporal (IMC) em ambos os grupos.

Recentemente, dois grandes ensaios clínicos aleatorizados controlados, o Diabetes Prevention Program 12 e o Finnish Diabetes Prevention Study 13, demonstraram a eficácia da intervenção do estilo de vida na prevenção primária do diabetes mellitus tipo 2 em portadores de TGD, sendo esta medida significativamente mais efetiva do que o tratamento medicamentoso.

Um dos estudos de maior destaque na literatura é o Diabetes Prevention Program 12. Ensaio clínico aleatorizado controlado conduzido nos Estados Unidos avaliou o impacto da intervenção de estilo de vida e tratamento medicamentoso em 3.234 adultos de origem multiétnica portadores de TGD e excesso de peso. Os voluntários foram recrutados através de divulgação na mídia, seguros de saúde, cartas e telefonemas e aleatoriamente alocados nos grupos "estilo de vida", "metformina" ou "placebo/controle”. O programa de intervenção no estilo de vida constou de 16 consultas individualizadas com um nutricionista nos primeiros seis meses e consultas mensais até o terceiro ano de seguimento do estudo, com sessões semanais de 
atividades físicas supervisionadas. Aos indivíduos com dificuldades de adesão ao programa de intervenção foram oferecidos equipamentos para a prática de exercícios e visitas domiciliares complementares 14 . Todos os participantes foram submetidos à avaliação clínica, bioquímica, antropométrica e de estilo de vida no início do estudo e após três anos com perda de seguimento de 7,5\%.

Os resultados obtidos no Diabetes Prevention Program foram surpreendentes, pois mesmo com uma perda de peso moderada (média de perda de peso de 0,$1 ; 2,1$ e 5,6kg nos grupos controle, metformina e estilo de vida, respectivamente) observou-se redução na incidência (IC95\%) de 58,0\% (48-66) dos casos de diabetes no grupo "estilo de vida”, sendo esta medida significativamente mais efetiva na prevenção primária do diabetes mellitus tipo 2 em relação ao uso da metformina [redução de incidência (IC95\%) 31,0\% (17-43)]. Após três anos de seguimento, a incidência observada foi de 11,0; 7,8 e 4,8 casos por 100 pessoas/ano nos grupos controle, metformina e estilo de vida, respectivamente. Neste estudo, 50,0\% dos indivíduos do grupo "estilo de vida" alcançaram a meta de $7,0 \%$ de perda de peso e $74,0 \%$ alcançaram a meta da prática de 150 minutos de atividades físicas semanais. Entretanto, os autores não relataram alterações do consumo alimentar habitual entre os participantes.

Outro estudo de grande impacto na literatura foi o Finnish Diabetes Prevention Study 13. Ensaio clínico aleatorizado controlado conduzido na Finlândia para a prevenção primária de diabetes mellitus tipo 2 avaliou o impacto da orientação de estilo de vida por um período médio de 3,2 anos em 522 adultos portadores de excesso de peso e TGD recrutados através divulgação na mídia e estratégias de rastreamento de portadores de fatores de risco. Os indivíduos foram aleatoriamente alocados entre os grupos "controle" e "estilo de vida", cujo programa de intervenção foi composto por sete consultas individualizadas com um nutricionista no primeiro ano e consultas trimestrais a partir do segundo ano do estudo. Além disso, os indivíduos participaram de um programa individualizado de práticas de atividades físicas com um professor de educação física ao longo do estudo. Atividades complementares, tais como aulas de culinária, visitas a supermercados para orientação de compras de alimentos e sessões de estudo em grupo, também foram oferecidas aos integrantes do grupo intervenção 15. Todos os participantes foram submetidos à avaliação clínica, bioquímica, antropométrica e de estilo de vida no início do estu- do e após 12 e 36 meses com perdas de seguimento de $10,0 \%$ e $17,0 \%$, respectivamente. No total, 43,0\% dos indivíduos do grupo intervenção atingiram a meta de perda de peso, $47,0 \%$ reduziram o consumo de gorduras totais, $26,0 \%$ atingiram a meta de consumo de gorduras saturadas e $25,0 \%$ dos indivíduos alcançaram a meta do consumo de fibras. Além disso, 86,0\% dos indivíduos relataram praticar quatro horas de exercícios por semana após três anos de seguimento.

Os resultados do estudo finlandês após três anos de seguimento demonstraram que a incidência de diabetes foi de 32 casos / 1.000 pessoas ao ano no grupo intervenção e de 78 casos/1.000 pessoas ao ano no grupo controle. A incidência de diabetes mellitus tipo 2 no grupo intervenção foi 58,0\% menor em relação ao observado no grupo controle [razão de azar (IC 95\%) de 0,4 (0,3-0,7)]. Após 12 meses de seguimento, a redução média (IC95\%) de peso corporal $[-4,7 \%(-5,0 ;-4,4)]$ e triglicérides séricos $[-18 \mathrm{mg} / \mathrm{dl}(-24 ;-12)]$ foram superiores ao observado no grupo controle $[-0,9 \%(-1,0 ;-0,8)] \mathrm{e}$ $[-1 \mathrm{mg} / \mathrm{dl}(-8 ; 6)]$, respectivamente. Contudo, não houve diferenças estatisticamente significantes entre os grupos para diferenças médias nos níveis séricos de colesterol total e frações após 12 meses de seguimento 15. Informações detalhadas de alteração do perfil de lipídios séricos após três anos de seguimento não foram relatadas.

Esses resultados demonstraram a eficácia da adoção de um estilo de vida saudável na redução da incidência do diabetes mellitus tipo 2 entre indivíduos com perfil elevado de risco. Estudos epidemiológicos mais recentes foram então conduzidos com o intuito de se avaliar o impacto de diferentes abordagens educacionais e intensidade de intervenção para promoção de qualidade de vida e controle de fatores de risco para o diabetes mellitus tipo 2 .

Um ensaio clínico aleatorizado controlado, conduzido na Nova Zelândia em 79 adultos normoglicêmicos portadores de resistência à insulina e excesso de peso, avaliou o impacto de intervenção nutricional intensiva ou moderada na melhoria da sensibilidade à insulina 16 . A população de estudo foi aleatoriamente alocada entre os grupos "controle" e grupos de intervenção de estilo de vida "intenso" ou "moderado”, com avaliação clínica, bioquímica, antropométrica e de estilo de vida no início do estudo e após quatro meses com perda de seguimento de $2,5 \%$. O programa de intervenção constou de consultas individualizadas de freqüência semanal durante todo o período de seguimento. Os resultados demonstraram au- 
mento em $23,0 \%$ na sensibilidade à insulina, estatisticamente significante apenas no grupo de intervenção intensiva de estilo de vida [aumento médio (IC95\%) de sensibilidade à insulina de $0,9(0,3-1,6)$ G.um-1.1-1]. Os valores médios (IC95\%) de diferença de peso nos grupos de intervenção "moderada" e "intensa" foram semelhantes: $-3,4(-5,4 ;-1,3)$ e $-4,7(-6,9 ;-2,4)$, respectivamente. Não se verificou alteração nos valores de lipídios séricos e glicemia de jejum após quatro meses de seguimento. Uma redução do consumo de gorduras saturadas foi observada em ambos os grupos de intervenção [média (IC95\%) de -7\% (-10; -5)]; porém, o aumento do consumo de fibras da dieta foi estatisticamente significante apenas no grupo de intervenção intensiva [média (IC95\%) 8,9 (2,9-14,9)] 16.

Um ensaio clínico aleatorizado controlado conduzido no Japão entre 173 homens adultos portadores de TGD avaliou o impacto de um programa educacional de estilo de vida saudável na redução de glicemia plasmática após um ano de acompanhamento 17. Os voluntários foram selecionados entre trabalhadores residentes na área metropolitana de Tóquio que apresentaram valores de glicemia duas horas após sobrecarga de glicose entre 180 e $200 \mathrm{mg} / \mathrm{dl}$. Os voluntários foram aleatoriamente alocados entre os grupos controle e intervenção, cujo programa constou de uma consulta individualizada com o nutricionista e orientações por escrito encaminhadas via correio. Todos os indivíduos foram submetidos à avaliação clínica, bioquímica, antropométrica e de estilo de vida no início do estudo e após um ano com perda de seguimento foi de $9,8 \%$. Os autores observaram redução percentual média (DP) na glicemia após sobrecarga de glicose maior entre os indivíduos do grupo intervenção [-8,2 $(1,9) \%] \mathrm{em}$ relação ao observado no grupo controle $[+11,2$ $(3,0) \%]$ após um ano de seguimento $(\mathrm{p}<0,05$, ANCOVA). Os autores não relataram as alterações de peso ou perfil lipídico após o período de intervenção 17.

O Study on Lifestyle Intervention and Impaired Glucose Tolerance Maastricht (SLIM) 18 é um ensaio clínico aleatorizado controlado em andamento com 102 adultos portadores de TGD e excesso de peso na Holanda. O objetivo principal é avaliar o impacto de um programa de intervenção no estilo de vida na tolerância à glicose e resistência à insulina entre portadores de fatores de risco. Os participantes, selecionados de um estudo de coorte 19 , foram aleatoriamente alocados entre os grupos "controle" e "intervenção no estilo de vida”, cujo programa constou de três consultas individualizadas com o nutricionista e uma sessão em grupo. To- dos os indivíduos foram submetidos à avaliação clínica, bioquímica, antropométrica e de estilo de vida no início do estudo e após 12 meses com perda de seguimento foi de $10,0 \%$. O desenho do estudo prevê uma duração de três anos. Após 12 meses de seguimento, houve diferenças estatisticamente significantes nos valores de glicemia pós-carga e HOMA no grupo intervenção [diferença média (DP) de -13,0 $(5,4)$ $\mathrm{mg} / \mathrm{dl}$ e de $-0,72(0,29)$, respectivamente] quando comparado ao grupo controle $[+0,2(0,3) \mathrm{mg} /$ $\mathrm{dl} \mathrm{e}+0,14(0,18)$, respectivamente]. As diferenças médias (DP) de IMC de $-0,9(0,2) \mathrm{kg} / \mathrm{m}^{2}$ (cerca de $-3,0 \%)$ e circunferência de cintura $[-3,5$ $(0,5)]$ foram superiores ao observado no grupo controle $\left[0,0(0,2) \mathrm{kg} / \mathrm{m}^{2} \mathrm{e}-1,4(0,6) \mathrm{cm}\right.$ de cintura, $\mathrm{p}<0,05$ segundo teste $t$ de Student $]$. Em relação ao consumo alimentar, uma redução de $21,0 \%$ CT de gorduras saturadas e um aumento de $18,0 \%$ CT no consumo de fibras foram observados no grupo intervenção, estatisticamente diferentes do observado no grupo controle (incremento médio de $4,3 \%$ e $7,6 \%$ no consumo de gorduras saturadas e fibras, respectivamente) Após três anos de seguimento será avaliada a incidência de diabetes mellitus tipo 2 entre os participantes 18 .

No Brasil, o único ensaio clínico aleatorizado controlado conduzido em adultos com o objetivo de avaliar o impacto da intervenção nutricional no controle de fatores de risco para o diabetes mellitus tipo 2 foi desenvolvido em uma unidade básica de saúde de São José do Rio Preto, São Paulo 20. Os voluntários, recrutados por meio de cartas encaminhadas a familiares de portadores de diabetes mellitus tipo 2 e divulgação na mídia, foram aleatoriamente alocados entre os grupos "controle" e "intervenção nutricional”. O programa de intervenção foi composto por três consultas individualizadas com o nutricionista durante os seis primeiros meses de seguimento. Todos os participantes foram submetidos à avaliação clínica, bioquímica, antropométrica e de estilo de vida no início do estudo e após 6 e 12 meses com perdas de seguimento de 20,0 e $30,0 \%$, respectivamente. Houve perda de peso estatisticamente significante no grupo intervenção [diferença média (IC95\%) -2,4 $(-3,2 ;-1,6) \mathrm{kg}]$ em relação ao grupo controle $[-0,3(-1,1 ;+0,4)]$, com redução de cerca de 15,0 e 12,0\% do LDL-c e colesterol total, respectivamente [diferença média LDL (IC95\%) -23,7(-38,9; -10,6) e colesterol total $-27,1(-44,5 ;-12,1)$ no grupo intervenção vs. $+2,9(-4,1 ;+8,3)$ LDL-c e $-0,3(-12,0 ;+10,7)$ para colesterol total grupo controle]. Os participantes foram reavaliados após um ano, observando-se manutenção da redução de peso e 
dos níveis séricos de LDL-c e colesterol entre os indivíduos do grupo intervenção. No entanto, não foram observadas diferenças na glicemia de jejum e pós-carga, em função, provavelmente, do pequeno número de participantes $(\mathrm{n}=104) 20$.

\section{Discussão}

As evidências científicas provenientes dos ensaios clínicos aleatorizados descritos no presente artigo sugerem a eficácia da intervenção nutricional no controle de fatores de risco e na prevenção primária do diabetes mellitus tipo 2 em adultos. Dos nove estudos analisados, cinco avaliaram o impacto das medidas na incidência da doença e quatro descreveram o efeito da intervenção nutricional no controle de fatores de risco para o diabetes mellitus tipo 2 , como consumo alimentar habitual, peso corporal e perfil de lipídios séricos.

Dentre os cinco estudos que investigaram o impacto das orientações nutricionais na incidência do diabetes mellitus tipo 2 9,10,11,12,13, dois estudos 9,11 não encontraram uma redução estatisticamente significante na ocorrência da doença. Por outro lado, houve uma redução de cerca de 30,0 a $60,0 \%$ nas taxas de progressão de TGD para diabetes mellitus tipo 2 entre adultos nos demais estudos avaliados 10,12,13. A principal hipótese de explicação para os controversos resultados pode estar relacionada a limitações metodológicas, pois tanto no estudo de Wing et al. 9 como na pesquisa publicada por Wein et al. 11 o tamanho amostral de $154 \mathrm{e}$ 200 participantes, respectivamente, foi muito pequeno para a avaliação de alterações na incidência do desfecho avaliado.

Nos estudos realizados nos Estados Unidos 12, na Finlândia 13 e China 10, apesar da moderada redução de peso observada (cerca de 3,5\%) associada a alterações do comportamento alimentar e prática de atividades físicas, o impacto na redução da incidência da doença (cerca de $50,0 \%$ ) entre indivíduos com excesso de peso e alterações do metabolismo da glicose (TGD) foi surpreendente. Entretanto, a estrutura e recursos empregados no Diabetes Prevention Program e no estudo finlandês não são adequados à realidade dos serviços de saúde pública de países em desenvolvimento dado seu alto custo. Por outro lado, no estudo chinês, a aleatorização dos indivíduos realizada por clínicas de tratamento limitou bastante a interpretação dos resultados.

Os demais ensaios clínicos aleatorizados descritos avaliaram o impacto das orientações nutricionais no controle dos fatores de risco para o diabetes mellitus tipo 2. Dentre os quatro artigos descritos, três relataram tempo de seguimento de um ano 17,18,20, e dois observaram uma redução de peso corporal nos indivíduos do grupo intervenção de cerca de $3,0 \%$ em relação ao início do estudo 18,20. No estudo conduzido na Nova Zelândia 16 , a maior redução média de peso observada $(5,4 \%$ e $6,9 \%$ nos grupos de intervenção moderada e intensiva, respectivamente) pode ser atribuída ao menor tempo de seguimento (quatro meses) quando a perda de peso pode ocorrer com maior intensidade. No trabalho publicado por Watanabe et al. 17, os autores não relataram a diferença de peso dos participantes após o período de intervenção. Segundo os resultados do Diabetes Prevention Program 12 e do estudo finlandês, uma perda de peso de cerca de $5,0 \%$ pode representar uma redução superior a 50,0\% no risco de desenvolver o diabetes mellitus tipo 2 entre indivíduos portadores de TGD, sugerindo que as medidas de intervenção adotadas nesses ensaios clínicos podem reduzir os riscos para DCNT.

O estudo conduzido na Austrália entre mulheres portadoras de TGD e história pregressa de diabetes mellitus tipo 2 gestacional foi o único que não observou mudança no consumo alimentar habitual e peso corporal após a intervenção no estilo de vida 11 . Neste estudo, cujo objetivo era avaliar o impacto de orientações de baixo custo, o programa de intervenção concentrou-se em orientações por escrito encaminhadas via correio, sugerindo que programas de maior intensidade são necessários para a promoção da saúde em adultos de elevado risco para diabetes mellitus tipo 2. No estudo japonês 17, que também utilizou como estratégia de intervenção orientações por escrito via correio, os autores não relataram qual foi a diferença de peso corporal observada. Por outro lado, apesar da maior perda de peso ter sido observada na pesquisa conduzida por Wing et al. 9, com uma redução de 9,0 e 10,0\% do peso corporal nos grupos "dieta" e "dieta e exercícios" após seis meses, esta medida apresentou menor impacto após 12 meses. No estudo de Wing et al. 9, as orientações nutricionais constaram de sessões em grupo com o nutricionista e houve uma redução da freqüência de encontros ao longo do estudo. Estes resultados sugerem que o atendimento individualizado com o nutricionista pode resultar em maior impacto na redução e manutenção do peso corporal de indivíduos com elevado risco metabólico, fato que pode ser fundamentado pelos resultados dos estudos conduzidos na Holanda 18 e Brasil 20.

O programa de intervenção de estilo de vida adotado no estudo brasileiro 20 também de- 
monstrou um impacto importante no perfil de lipídios séricos dos indivíduos avaliados, com uma redução de média de LDL-c (cerca de 15,0\% após 6 e 12 meses de seguimento) superior ao observado no estudo de Wing et al. 9 em que a redução média foi de $10,0 \%$ após seis meses, sendo três vezes superior à redução média observada em ensaios clínicos aleatorizados controlados com intervenção nutricional para o controle de dislipidemia (média de 5,0\%) 21. Além disso, no estudo brasileiro houve a manutenção dos níveis de HDL-c.

Em relação aos valores de glicemia, com exceção dos estudos que avaliaram a redução da incidência do diabetes mellitus tipo 2 com maior tempo de seguimento, apenas no estudo japonês 17 houve uma redução estatisticamente significante na glicemia duas horas após sobrecarga de glicose em período relativamente curto de tempo. Esses resultados sugerem a necessidade de um maior tempo de seguimento para a detecção de diferenças nos valores de glicemia em estudos epidemiológicos.

Dentre todos os estudos descritos, com exceção dos estudos de Sartorelli et al. 20 e Wing et al. 9, a população alvo consistiu exclusivamente de indivíduos portadores de TGD, nos quais as medidas de intervenção poderão ser tardias na prevenção do diabetes mellitus tipo 222 . Além disso, os indivíduos com perfil metabólico comprometido podem apresentar diferentes respostas fisiológicas e de adesão ao tratamento dietético, limitando a extrapolação dos resultados de todos os estudos para a comunidade em geral.

As perdas de seguimento dos participantes após um ano de intervenção variaram de $2,5 \%$ no estudo conduzido na Nova Zelândia 16 a $30,0 \%$ observadas no estudo brasileiro 20 , sugerindo que a intensidade do tratamento e a freqüência das consultas podem influenciar a adesão ao programa de intervenção.

Segundo a Associação Americana de Diabetes 23 , estudos de baixo custo, adequados a condições usuais de serviços públicos de saúde, que avaliem a manutenção de peso e da prática de atividades físicas após a interrupção do tratamento são imprescindíveis para a avaliação da real eficácia de programas de prevenção primária de diabetes mellitus tipo 2 destinados à comunidade. Dentre todos os estudos descritos no presente artigo de revisão, apenas dois foram desenvolvidos em países em desenvolvimento: China e Brasil. Estima-se que nos próximos vinte anos o maior número de casos novos de diabetes mellitus tipo 2 seja registrado em adultos jovens residentes de países expostos a alterações de estilo de vida 1 . No Brasil, modificações no consumo alimentar, estilo de vida sedentário e conseqüente aumento da freqüência do excesso de peso, associado ao envelhecimento populacional, têm sido apontados como possíveis fatores envolvidos nas elevadas taxas de diabetes mellitus tipo $2 \mathrm{ob}$ servadas no Brasil 24, ressaltando a relevância da implementação de programas de prevenção primária de diabetes mellitus tipo 2 em serviços públicos de saúde.

\section{Conclusão}

Os resultados de ensaios clínicos aleatorizados conduzidos em indivíduos portadores de fatores de risco fornecem evidências sobre a eficácia da intervenção no estilo de vida - com ênfase na alimentação saudável e prática de atividades físicas - na redução do risco para o diabetes mellitus tipo 2 , sendo tais medidas significativamente mais eficazes que a intervenção medicamentosa.

A influência da carga genética no desenvolvimento da doença é indiscutível. Todavia, a prevenção ou retardo do desenvolvimento do diabetes mellitus tipo 2 entre indivíduos portadores de fatores de risco possui um impacto relevante na qualidade de vida da população, com redução de custos em saúde pública com complicações decorrentes da evolução da doença.

Os dados provenientes de países em desenvolvimento são escassos, mas os estudos disponíveis sugerem um importante impacto na melhoria da qualidade de vida de indivíduos com elevado risco metabólico por meio de medidas simples de intervenção adaptadas às condições usuais de unidades básicas de saúde.

Entretanto, conforme recomendação da OMS 5 em recente publicação sobre prevenção de doenças crônicas, a implementação de programas de mudança de estilo de vida em indivíduos portadores de fatores de risco deve ser associada a alterações ambientais que favoreçam as escolhas individuais na adoção e manutenção do estilo de vida saudável. Além disso, o estímulo à valorização cultural pela saúde constitui uma ferramenta de fundamental importância no alcance das metas do estilo de vida saudável. 


\section{Resumo}

O diabetes mellitus tipo 2 pode ser considerado um dos principais problemas de saúde pública no mundo atual. A prevalência crescente em diversos países e o difícil controle metabólico dos indivíduos com a doença em evolução justificam o estudo de estratégias para prevenção primária do diabetes mellitus tipo 2. Ensaios clínicos aleatorizados controlados recentes sugerem um impacto importante da intervenção nutricional na redução do risco de desenvolvimento de diabetes mellitus tipo 2 entre indivíduos portadores de fatores de risco. O presente artigo de revisão descreve o impacto de programas de intervenção no estilo de vida em ensaios clínicos aleatorizados controlados para a prevenção primária do diabetes mellitus tipo 2.

Diabetes Mellitus Tipo II; Terapia Nutricional; Doença Crônica

\section{Colaboradores}

D. S. Sartorelli realizou o levantamento bibliográfico. L. J. Franco contribuiu na interpretação dos dados e revisão do manuscrito. M. A. Cardoso e D. S. Sartorelli analisaram os dados e redigiram o manuscrito. M. A. Cardoso realizou a revisão do manuscrito.

\section{Referências}

1. King H, Aubert RE, Herman WH. Global burden of diabetes, 1995 - 2025. Diabetes Care 1998; 21:1414-31.

2. Donahue RP, Orchard TJ. Diabetes mellitus and macrovascular complications: an epidemiological perspective. Diabetes Care 1992; 15:1141-55.

3. Assunção MCF, Santos IS, Costa JSD. Avaliação do processo da atenção médica: adequação do tratamento de pacientes com diabetes mellitus, Pelotas, Rio Grande do Sul, Brasil. Cad Saúde Pública 2002; 18:205-11.

4. Assunção MCF, Santos IS, Gigante D. Atenção primária em diabetes no Sul do Brasil: estrutura, processo e resultado. Rev Saúde Pública 2001; 35:88-95.

5. Joint WHO/FAO Expert Consultation. Diet, nutrition and the prevention of chronic diseases. Geneva: World Health Organization/Food and Agriculture Organization; 2003.

6. Gimeno SGA, Ferreira SRG, Franco LJ, Hirai AT, Matsumura L, Moisés RS, et al. Prevalence and 7year incidence of type 2 diabetes mellitus in a Japanese-Brazilian population: an alarming public health problem. Diabetologia 2003; 45:16358.

7. Hu FB, Manson JE, Stampfer MJ, Colditz G, Liu S, Solomon CG, et al. Diet, lifestyle and the risk of type 2 diabetes mellitus in women. N Engl J Med 2001; 345:790-7.

8. Erikson KF, Lindgard F. No excess 12-year mortality in men with impaired glucose tolerance who participated in the Malmö Preventive Trial with diet and exercise. Diabetologia 1998; 41:1010-6.

9. Wing RR, Venditti E, Jakicic JM, Polley BA, Lang W. Lifestyle intervention in overweight individual with a family history of diabetes. Diabetes Care 1998; 21:350-9.

10. Pan XR, Li GW, Hu YH, Wang JX, Yang WY, An ZX, et al. Effect of diet and exercise in preventing NIDDM in people with impaired glucose tolerance. Diabetes Care 1997; 20:537-44.

11. Wein P, Beischer N, Harris C, Permezel M. A trial of simple versus intensified dietary modification for prevention of progression to diabetes mellitus in women with impaired glucose tolerance. Aust N Z J Obstet Gyneacol 1999; 39:162-6.

12. Diabetes Prevention Program Research Group. Reduction in the incidence of type 2 diabetes with lifestyle intervention or metformin. $\mathrm{N}$ Engl J Med 2002; 346:393-403.

13. Tuomilehto J, Lindstrom J, Eriksson JG, Valle TT, Hamalainen H, Ilanne-Parikka P, et al. Prevention of type 2 diabetes mellitus by changes in lifestyle among subjects with impaired glucose tolerance. N Engl J Med 2001; 344:1343-50.

14. Diabetes Prevention Program Research Group. The diabetes prevention program (DPP): description of lifestyle intervention. Diabetes Care 2002; 25:2165-71.

15. Lindstrom J, Louheranta A, Mannelin M, Rastas M, Salminen V, Eriksson J, et al. The Finnish diabetes prevention study (DPS). Lifestyle intervention and 3-year results on diet and physical activity. Diabetes Care 2003; 26:3230-6. 
16. McAuley KA, Williams SM, Mann JI, Gouling A, Chisholm A, Wilson N, et al. Intensive lifestyle changes are necessary to improve insulin sensitivity. Diabetes Care 2002; 25:445-52.

17. Watanabe M, Yamaoka K, Yokotsuka M, Tango T. Randomized controlled trial of a new dietary education program to prevent type 2 diabetes in a high-risk group of Japanese male workers. Diabetes Care 2003; 26:3209-14.

18. Mensink M, Feskens EJM, Saris WHS, Bruin TWA, Blaak EE. Study on lifestyle intervention and impaired glucose tolerance Maastricht (SLIM): preliminary results after one year. Int J Obes 2003; 27:377-84.

19. VanDam RM, Boer JM, Feskens EJ, Deidell JC. Parental history of diabetes modifies the association between abdominal adiposity and hyperglycemia. Diabetes Care 2001; 24:1454-9.
20. Sartorelli DS, Sciarra EC, Franco LJ, Cardoso MA. Primary prevention of type 2 diabetes through nutritional counseling. Diabetes Care 2004; 27: 3019.

21. Tang JL, Armitage JM, Lancaster T, Silagy CA, Fowler GH, Neil HAW. Systematic review of dietary intervention trials to lower blood total cholesterol in free-living subjects. BMJ 1998; 316:121320.

22. Mann JI. Diet and risk of coronary heart disease and type 2 diabetes. Lancet 2002; 360:783-9.

23. American Diabetes Association/National Institute of Diabetes, Digestive and Kidney Disease. The prevention or delay of type 2 diabetes. Diabetes Care 2002; 25:742-9.

24. Sartorelli DS, Franco LJ. Tendências do diabetes mellitus no Brasil: o papel da transição nutricional. Cad Saúde Pública 2003, 19 Suppl 1:29-36.

Recebido em 22/Set/2004

Versão final reapresentada em 15/Jun/2005

Aprovado em 06/Jul/2005 